\title{
Artifacts in X-ray Dark-Field Tomography
}

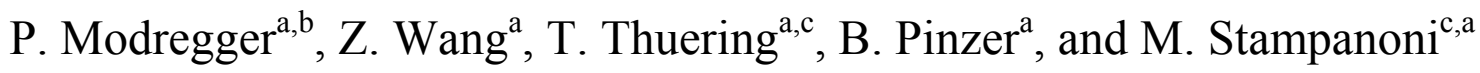 \\ ${ }^{a}$ Swiss Light Source, Paul Scherrer Institut, 5232 Villigen PSI, Switzerland \\ ${ }^{b}$ Center for Biomedical Imaging, 1015 Lausanne, Switzerland \\ ${ }^{c}$ Institute for Biomedical Engineering, University and ETH Zurich, 8092 Zurich, Switzerland
}

\begin{abstract}
Grating-based x-ray imaging provides three principle kinds of contrast: absorption, phase, and dark-field. Due to the availability of tomographic reconstruction algorithms for the dark-field contrast, it is now possible to take advantage of quantitative scatter information. However, the published algorithm is based on several assumptions that might be violated in reality. We use numerical simulations in order to identify artifacts in the reconstructions, which is crucial for the interpretation of experimental data.
\end{abstract}

Keywords: X-ray imaging, dark-field tomography, artifacts

PACS: 87.59.-e, 78.70.Ck, 87.57.Q-

\section{INTRODUCTION}

Grating interferometry is a recently established phase-sensitive x-ray imaging technique that provides a particular high sensitivity towards density variations in the sample [1]. In addition to the absorption and phase contrast, a grating interferometer also delivers complementary information on local scattering strength in the sample, which is also reported as dark-field image [2]. Since elastic scattering may be regarded as unresolved local phase variations, the scattering signal provides information on a length scale smaller than the pixel size of the utilized detector.

The GI (see Fig. 1) utilizes a phase grating and an absorption grating downstream of the sample in order to retrieve the contributions of absorption, phase, and dark-field signal. This is achieved by laterally scanning one of the gratings in steps of a fraction of the grating's pitch and acquiring an image for each step. Therewith, a so-called phase stepping curve (PSC) is obtained for each pixel, which can then be analyzed in order to yield the three different kinds of contrast.

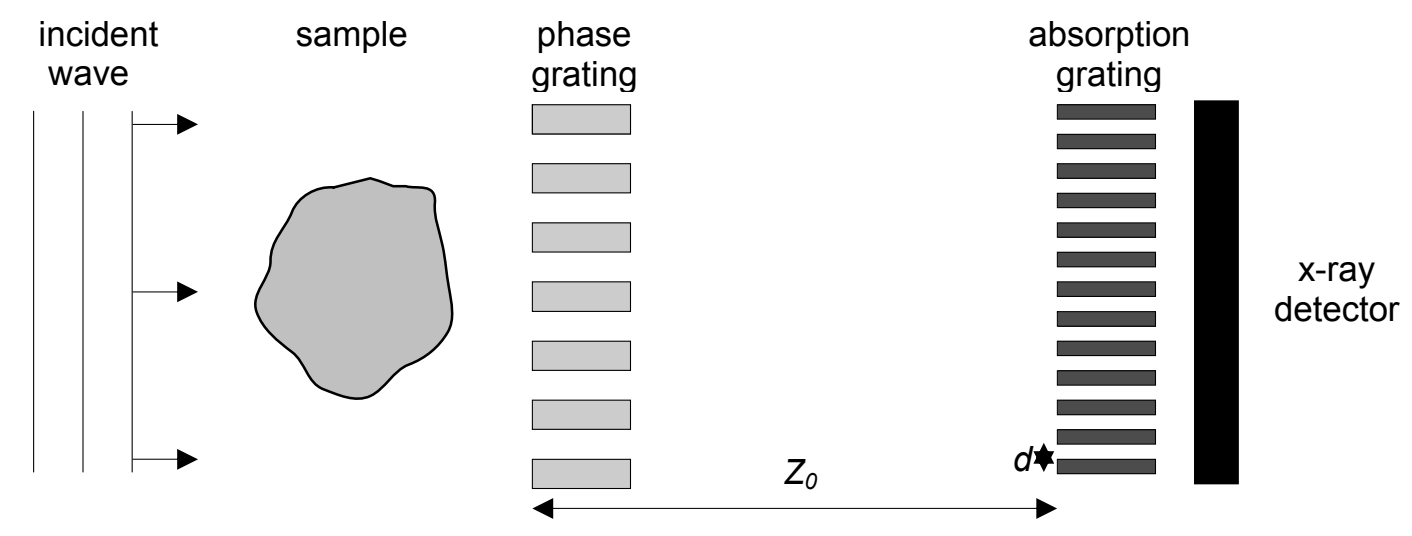

FIGURE 1. Sketch of the grating interferometer (GI).

The 10th International Conference on X-ray Microscopy AIP Conf. Proc. 1365, 269-272 (2011); doi: 10.1063/1.3625356 (C) 2011 American Institute of Physics 978-0-7354-0925-5/\$30.00 
Based on a theoretical description of a small scattering contribution to the image formation process [3], Wang et al. established a first approach to quantitative dark-field tomography with the grating interferometer [4]. However, this approach is based on several approximations, and the artifacts that arise from violations of these approximations are still unknown. Therefore, we used numerical simulations in order to identify the artifacts, which is crucial for the interpretation of experimental data.

\section{THEORY}

Originally developed for application to analyzer-based imaging [3], Kelashvili et al. developed a radiative transport model that is independent of the utilized imaging method [4]. The local scattering strength in the sample $\sigma_{s}(x, z)$ is connected to the total scattering width $\sigma_{d e t}$ at the position of the detector. It was shown that $\sigma_{d e t}$ fulfills the line integral

$$
\sigma_{d e t}^{2}=\int d z \frac{\rho(z) \sigma_{s}(x, z)}{2 \alpha_{p}},
$$

where the density of the sample is denoted by $\rho$ and the scatter strength of a single object point by $\alpha_{p}$. Thus, standard filtered-back projection [4] is applicable to scattering, which opens the possibility for quantitative darkfield tomography.

Wang et al. applied this general description to grating interferometry [5]. Here, the visibility $V$ of the PSCs constitute the dark-field signal, which may be obtained by Fourier transform and calculating the relation:

$$
V_{i}=\frac{\hat{P}_{i}\left(q_{1}\right)}{P_{i}\left(q_{0}\right)} \text { with } i=\text { flat } \text { or sample. }
$$

The hat symbol ( ${ }^{\wedge}$ ) indicates the Fourier transform, the zero frequency component is denoted by $q_{0}$, and the first harmonic of the periodic PSC by $q_{1}$. The index $i$ distinguishes between the PSC acquired with sample and without (i.e., flat image). With the assumption of a Gaussian-shaped scatter angle distribution, the visibility of the PSC is then related to the scatter width of the beam by

$$
\sigma_{\text {exp }}^{2}=\frac{-1}{2 \pi}\left(\frac{d}{z_{o}}\right)^{2} \ln \frac{V_{\text {sample }}}{V_{\text {flat }}},
$$

with $d$ the pitch of the absorption grating and $z_{o}$ the inter-grating distance. The equation $\sigma_{e x p}^{2}=\sigma_{d e t}^{2}$ then constitutes a Fourier slice theorem for dark-field tomography.

During the derivation several assumptions were performed that might not apply in reality. First, it is assumed that the scatter signal of each sample feature is not spread over more than one pixel of the detector. Secondly, the scatter signal is assumed to be Gaussian-shaped. Since the central limit theorem of probability theory applies, a Gaussian for the final scattering distribution at the detector will be a very good approximation in most cases. However, it seems very unlikely that the same holds true for all the sample features. Finally, it is implicitly assumed that the scatter strength $\sigma_{s}(x, z)$ does not depend on the direction of illumination. But for highly ordered structures (a crystal might be regarded as an extreme case), the assumption of anisotropic scattering is not realistic. Thus, it seems appropriate to identify and classify artifacts that arise due to violations of these assumptions.

\section{NUMERICAL SIMULATIONS}

In the introduction it was stated that the dark-field signal may be regarded as the width of the unresolved scatter angle distribution $g(\alpha)$ within a detector pixel. We will use this idea for the development of a simple yet general framework for the numerical simulation of dark-field tomography.

Starting with an arbitrary image representing the refractive index decrement $\delta$ of a model sample, we calculate the Radon transform (i.e., the sinogram) and the first derivative thereof. The result is the refraction angle introduced by the sample into the beam as a function of the rotation angle. Several adjacent pixels are then merged in order to form the scatter signal $g(\alpha)$ within a detector pixel. According to [2], the PSC with sample is then given by the convolution of $g(\alpha)$ with the flat-field PSC. Finally, the Fourier-based analysis procedure described above is applied 
to the PSCs for retrieving the dark-field signal. Note that in general the scatter angle distribution will not be of Gaussian shape as required.

Anisotropic scattering is included into the numerical simulation by artificially adjusting $\delta$ during the rotation of the input image in the Radon transform.

This procedure provides the dark-field sinogram, which is then used as input for tomographic reconstruction as described in the section above, yielding the numerically simulated dark-field slice. In order to have a mean for comparison, we processed the original as follows. Variations on a small length scale of the original image correspond to scattering. Thus, the scattering strength of a particular region in the model sample can be attributed by the standard deviation of the refractive decrement in this region. Thus, the image of a real standard deviation is taken for comparison and called the 'original slice.'

First, we investigated the effect of violating the assumption of a Gaussian-shaped scatter angle distribution. The well-known Shepp-Logan phantom [6] serves as a model sample. Figure 2 compares the original to the reconstructed slice. While all sample features are reconstructed, some show negative scatter-width values (dark areas in the image). Thus, it may be concluded that strong deviation of the scatter distribution from a Gaussian shape results into physically meaningless scatter-width values.

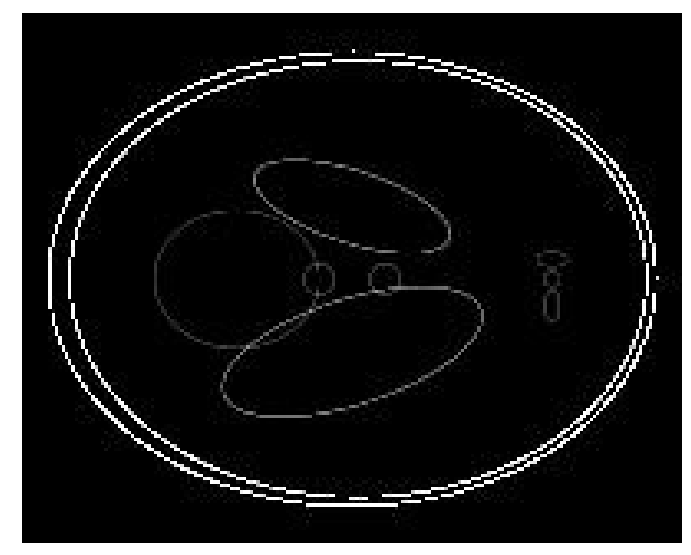

(a) original slice

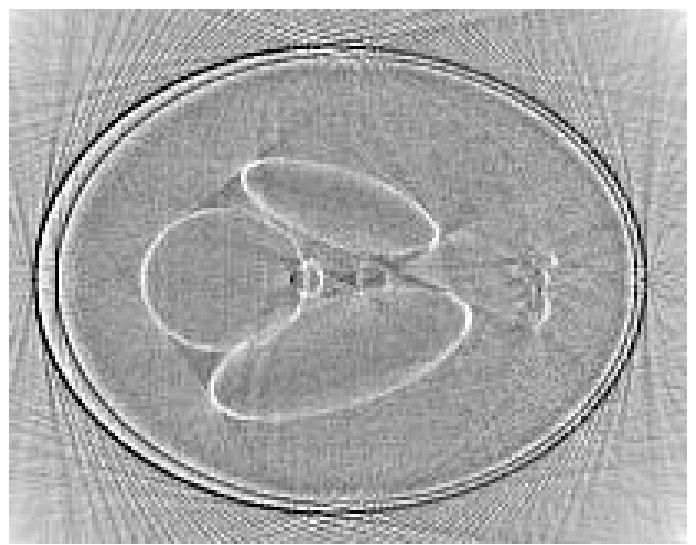

(b) reconstructed slice

FIGURE 2. Artifacts due to violation of the assumption that the scattering is Gaussian-shaped. Gray values correspond to local scatter strength.

Secondly, a model sample consisting of three circles was used, where the scatter width of two of the circles was set to oscillate during rotation. Thus, both assumptions of isotropic scattering as well as Gaussian-shaped scatter distribution were violated in this example. Figure 3 shows that a disappearance of sample features as well as additional streak artifacts may be observed if the scattering of the sample is indeed anisotropic.

\section{CONCLUSION}

In conclusion, we described a simple but general approach to numerically simulate dark-field tomography in grating interferometry, which takes into account anisotropic scattering as well as an arbitrary scattering distribution within a sample feature. This numerical framework was used to investigate the limits of the reconstruction algorithm for quantitative dark-field tomography developed by Kelashvili et al. and Wang et al.

The violation of two assumptions for this algorithm was studied. First, a non-Gaussian scattering distribution leads to negative scattering widths, which have no physical meaning. Secondly, anisotropic scattering produces an inhomogeneous disappearance of sample features in the reconstructed slices. Although, both kinds of artifacts may not be readily distinguishable from other occurring artifacts (e.g., involuntary sample movement, misalignment), this identification allows one to assess probable attributes of the sample. If, for example, some sample features in the reconstructed slices are conspicuously absent, this may be taken as a strong indication of anisotropic scattering. 


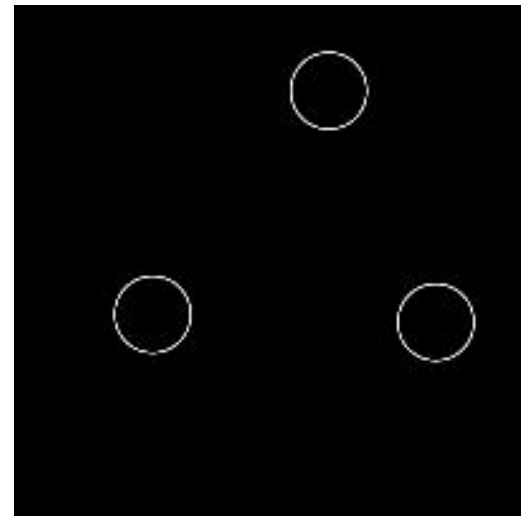

(a) original slice with isotropic scattering seen at $25^{\circ}$

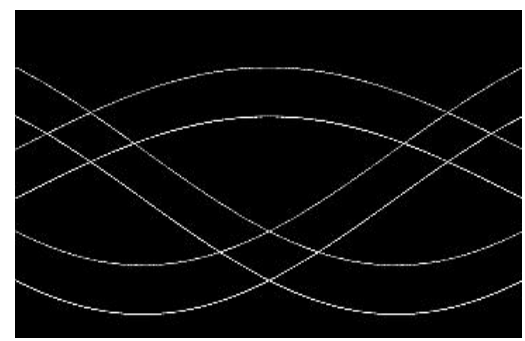

(c) dark-field sinogram with isotropic scattering

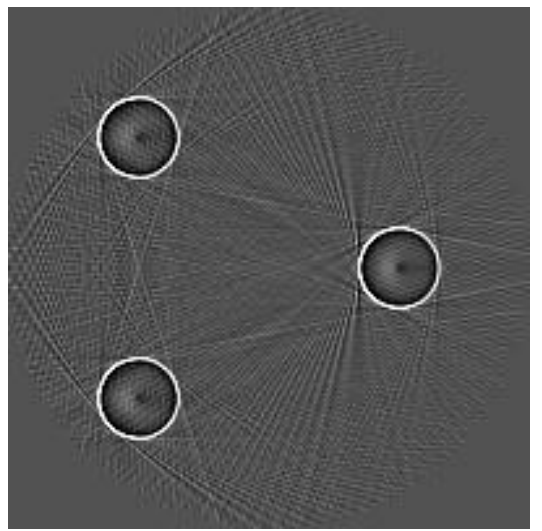

(e) reconstructed slice with isotropic scattering

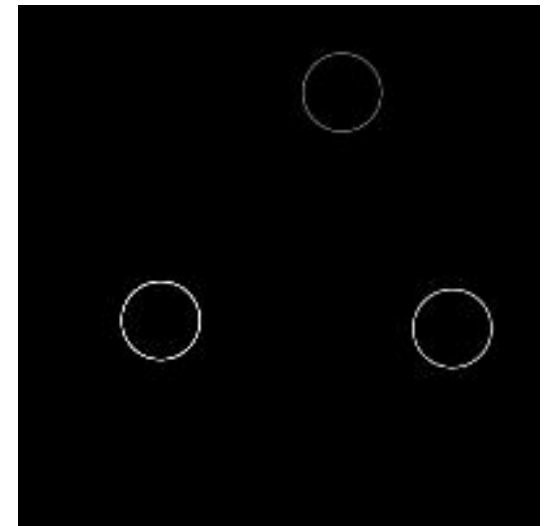

(b) original slice with anisotropic scattering seen at $25^{\circ}$

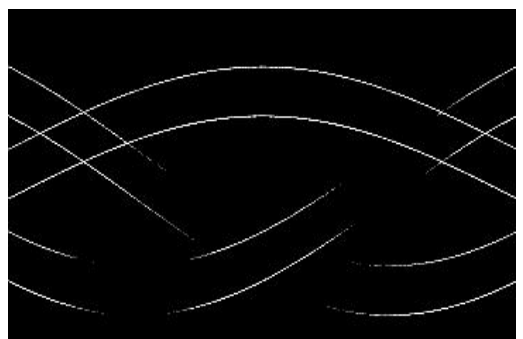

(d) dark-field sinogram with anisotropic scattering

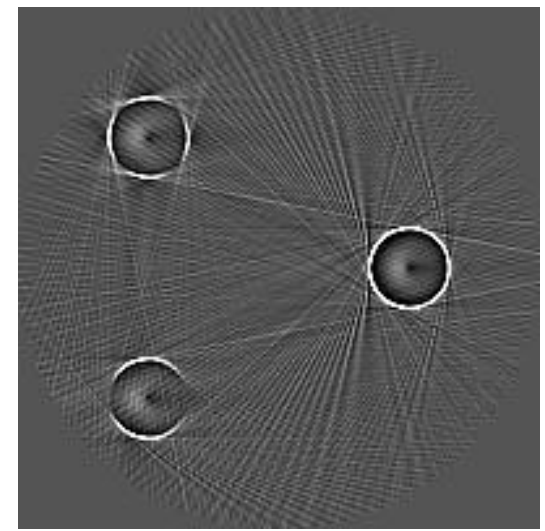

(f) reconstructed slice with anisotropic scattering

FIGURE 3. Artifacts due to violation of the assumption of anisotropic scattering. Gray values correspond to local scatter strength. On the left-hand side the scattering was set to be isotropic, serving as a reference. On the right-hand side the scattering of two circles was set to vary with the illumination direction, which is clearly visible in the sinogram. The disappearance of sample features is obvious.

\section{REFERENCES}

1. T. Weitkamp et al., Opt. Express 13, 6296 (2005).

2. F. Pfeiffer et al., Nature Materials 7, 134 (2008).

3. D. Chapman et al., Phys. Med. Biol. 42, 2015 (1997).

4. G. Khelashvili et al., Phys. Med. Biol. 51, 221 (2006).

5. Z. Wang, K. Kang, Z. Huang, and Z. Chen, Appl. Phys. Lett. 95, 094105 (2009).

6. A. C. Kak and M. Slaney, Principles of Computerized Tomographic Imaging, IEEE Press, 1988. 